\title{
A Bivariate Threshold Time Series Model for Analyzing Australian Interest Rates
}

\author{
W.S. Chan and S.H. Cheung

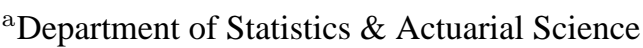 \\ The University of Hong Kong \\ Hong Kong, PR China \\ ${ }^{\mathrm{b}}$ Department of Statistics \\ The Chinese University of Hong Kong \\ Hong Kong, PR China
}

\begin{abstract}
In recent years, research in nonlinear time series analysis has grown rapidly. Substantial empirical evidence of nonlinearities in economic time series fluctuations has been reported in the literature. Nonlinear time series models have the advantage of being able to capture asymmetries, jumps, and time irreversibility which are characteristics of many observed financial and economic time series. As compared to the linear models, the nonlinear time series models provide a much wider spectrum of possible dynamics for the economic time series data. In this paper, we explore the use of nonlinear time series models to analyze Australian interest rates. In particular, we concentrate on the class of bivariate threshold autoregressive (BTAR) models. Monthly Australian interest rates from 1957.1 to 2002.8 are considered. The series under study are 2-year and 15-year government bonds, representing short-term and long-term series in the term structure of interest rates. A BTAR model is fitted to the observed vector series and the results show that the dynamic structure of the two interest rate series depends heavily on the status (expansion versus contraction) of the economy.
\end{abstract}

Keywords: Arranged Autoregression; Bivariate time series; Model Change; Nonlinearity test

\section{INTRODUCTION}

The class of threshold autoregressive (TAR) models (Tong, 1978 and 1983) has been widely employed in the literature to explain various empirical nonlinear phenomena observed in economic time series. See, e.g., Krag̈er and Kugler (1993), Peel and Speight (1994) and Chappell et al. (1996) for foreign exchange rate variables; Yadav et al. (1994) for futures market; Tiao and Tsay (1994) and Potter (1995) for US GNP; Montgomery et al. (1998) for US unemployment; and De Gooijer and Vidiella-iAnguera (2003) for monthly inflation rates.

The size and the level of sophistication of the market of fixed income securities around the world increased dramatically over the last 10 years and it became a prime test bed for financial institutions and academic research. Interest rate models are crucial to financial economists to price derivatives such as swaps, quantify and manage financial risk, and set monetary policy. Actuaries are also being called upon to incorporate interest rate models in a variety of applications, including dynamic financial analysis (DFA), ratemaking, and valuation (Wilkie, 1995).
In this paper, we explore the use of nonlinear threshold models to analyze Australian interest rates. In particular, we concentrate on the class of bivariate threshold autoregressive (BTAR) models. Monthly Australian interest rates from 1957.1 to 2002.8 are considered. The series under study are 2-year and 15-year government bonds, representing short-term and long-term series in the term structure of interest rates. A BTAR model is fitted to the observed vector series and the results show that the dynamic structure of the two interest rate series depends heavily on the status (expansion versus contraction) of the economy.

The paper proceeds as follows. Section 2 provides a brief review on BTAR modelling. Section 3 presents the empirical results. Discussion and conclusion follow in the final section.

\section{BIVARIATE TAR MODELS}

\subsection{The Model}

Tsay (1998) generalizes the univariate threshold principle to a multivariate framework. In this article, we consider a bivariate time series $\mathbf{Y}_{t}=\left(y_{1 t}, y_{2 t}\right)^{\prime}$. A $k$-regime $\operatorname{BTAR}\left(d ; p_{1}, \ldots, p_{k}\right)$ model is defined 


$$
\mathbf{Y}_{t}=\left\{\begin{array}{cc}
\mathbf{C}_{0}^{(1)}+\sum_{j=1}^{p_{1}} \boldsymbol{\Phi}_{j}^{(1)} \mathbf{Y}_{t-j}+\boldsymbol{\varepsilon}_{t}^{(1)}, & \text { if } z_{t-d} \leq r_{1} \\
\mathbf{C}_{0}^{(2)}+\sum_{j=1}^{p_{2}} \boldsymbol{\Phi}_{j}^{(2)} \mathbf{Y}_{t-j}+\boldsymbol{\varepsilon}_{t}^{(2)}, & \text { if } r_{1}<z_{t-d} \leq r_{2} \\
\vdots & \vdots \\
\mathbf{C}_{0}^{(k)}+\sum_{j=1}^{p_{k}} \boldsymbol{\Phi}_{j}^{(k)} \mathbf{Y}_{t-j}+\boldsymbol{\varepsilon}_{t}^{(k)}, & \text { if } r_{k-1}<z_{t-d}
\end{array}\right.
$$

where $k$ is the number of regimes in the model, $d$ is the delay parameter, $p_{i}$ is the autoregressive order in the $i$ th regime of the model, $\mathbf{C}_{0}^{(i)}$ are $(2 \times 1)$-dimensional constant vectors and $\boldsymbol{\Phi}_{j}^{(i)}$ are $(2 \times 2)$-dimensional matrix parameters for $i=$ $1, \ldots, k$. The threshold parameters satisfy the constraint $-\infty=r_{0}<r_{1}<r_{2}<\ldots<r_{k-1}<r_{k}=$ $\infty$. The innovational vectors in the $i$ th regime satisfy $\varepsilon_{t}^{(i)}=\boldsymbol{\Sigma}_{i}^{1 / 2} \mathbf{a}_{t}$, where $\boldsymbol{\Sigma}_{i}^{1 / 2}$ are symmetric positive definite matrices and $\left\{\mathbf{a}_{t}\right\}$ is a sequence of serially uncorrelated normal random vectors with mean $\mathbf{0}$ and covariance matrix $\mathbf{I}$, the $(2 \times 2)$-dimensional identity matrix. The threshold variable $z_{t-d}$ is assumed to be stationary, and it depends on the observable past history of $\mathbf{Y}_{t-d}$. For example, we can set

$$
z_{t-d}=\boldsymbol{\omega}^{\prime} \mathbf{Y}_{t-d}
$$

where $\boldsymbol{\omega}$ is a pre-specified $(2 \times 1)$-dimensional vector. When $\boldsymbol{\omega}=(1,0)^{\prime}$, the threshold variable is simply $z_{t-d}=y_{1, t-d}$. When $\boldsymbol{\omega}=\left(\frac{1}{2}, \frac{1}{2}\right)^{\prime}$, the threshold variable is the average of the two elements in $\mathbf{Y}_{t-d}$.

\subsection{Modeling Procedures}

Analogous to the Tsay (1989) procedures for univariate TAR modeling, Tsay (1998) extends the method to multivariate situation. The method has been applied successfully to many datasets, ranging from U.S. interest rates to Icelandic river flow series. This article uses the Tsay (1998) strategy for BTAR modeling.

\section{Testing for Nonlinearity}

Given $p=\max \left\{p_{1}, \ldots, p_{k}\right\}$ and $d \leq p$, we observe the bivariate vector time series $\left\{\mathbf{Y}_{1}, \ldots, \mathbf{Y}_{n}\right\}$. It should be noted that the threshold variable $z_{t-d}$ in (1) can only assume values in $\mathcal{Z}=$ $\left\{z_{p+1-d}, \ldots, z_{n-d}\right\}$. Let $(i)$ be the time-index of the $i$ th smallest observation in $\mathcal{Z}$. Tsay (1998) considers the multivariate generalization of the ordered regression arrangement. Rolling ordered bivariate

$$
\begin{aligned}
\left(\begin{array}{c}
\mathbf{Y}_{(1)+d}^{\prime} \\
\mathbf{Y}_{(2)+d}^{\prime} \\
\vdots \\
\mathbf{Y}_{(j)+d}^{\prime}
\end{array}\right)= & \left(\begin{array}{cccc}
1 & \mathbf{Y}_{(1)+d-1}^{\prime} & \ldots & \mathbf{Y}_{(1)+d-p}^{\prime} \\
1 & \mathbf{Y}_{(2)+d-1}^{\prime} & \ldots & \mathbf{Y}_{(2)+d-p}^{\prime} \\
\vdots & \vdots & \ddots & \vdots \\
1 & \mathbf{Y}_{(j)+d-1}^{\prime} & \ldots & \mathbf{Y}_{(j)+d-p}^{\prime}
\end{array}\right) \\
& \times\left(\begin{array}{c}
\mathbf{C}_{0}^{\prime} \\
\mathbf{\Phi}_{1}^{\prime} \\
\vdots \\
\mathbf{\Phi}_{p}^{\prime}
\end{array}\right)+\left(\begin{array}{c}
\boldsymbol{\varepsilon}_{(1)+d}^{\prime} \\
\boldsymbol{\varepsilon}_{(2)+d}^{\prime} \\
\vdots \\
\boldsymbol{\varepsilon}_{(j)+d}^{\prime}
\end{array}\right)
\end{aligned}
$$

can be arranged successively, where $j=m, m+$ $1, \ldots, n-p$, and $m$ is the number of startup observations in the ordered autoregression. Tsay (1998) suggests a range of $m$ (between $3 \sqrt{n}$ and $5 \sqrt{n}$ ). Different values of $m$ can be used to investigate the sensitivity of the modeling results with respect to the choice. It should be noted that the ordered autoregressions are sorted by the variable $z_{t-d}$, which is the regime indicator in the BTAR model.

Let $\widehat{\mathbf{e}}_{(m+1)+d}$ denote the one-step-ahead standardized predictive residual from the above least squares fitted multivariate regression for $j=m$. Tsay (1998, p. 1190) provides the direct computational formula for $\widehat{\mathbf{e}}_{(m+1)+d}$. Alternatively, they can be easily obtained from many commonly used statistical software packages (e.g., Timm and Mieczkowski, 1997). Analogous to the univariate case, if the underlying model is a linear vector autoregressive process, then the predictive residuals are white noise and they are uncorrelated to the regressor $\mathbf{X}_{t}^{\prime}=\left\{1, \mathbf{Y}_{t-1}^{\prime}, \mathbf{Y}_{t-2}^{\prime}, \ldots, \mathbf{Y}_{t-p}^{\prime}\right\}$. However, if $\mathbf{Y}_{t}$ follows a threshold process, then the predictive residuals are correlated with the regressor. Tsay (1998) utilizes this property and considers the multivariate regression

$$
\widehat{\mathbf{e}}_{(l)+d}^{\prime}=\mathbf{X}_{(l)+d}^{\prime} \boldsymbol{\beta}+\mathbf{w}_{(l)+d}^{\prime}
$$

for $l=m+1, \ldots, n-p$ with $\boldsymbol{\beta}$ is the matrix regression parameter and $\mathbf{w}_{(l)+d}^{\prime}$ is the matrix of residuals. The problem of testing nonlinearity is then transformed to testing of the hypothesis $H_{0}: \boldsymbol{\beta}=\mathbf{0}$ in the above regression. Tsay (1998) employs the test statistic

$$
C(d)=(n-p-m-k p-1) \times\left\{\ln \left|\mathbf{S}_{\mathbf{0}}\right|-\ln \left|\mathbf{S}_{\mathbf{1}}\right|\right\}
$$

where $|\mathbf{A}|$ denotes the determinant of the matrix $\mathbf{A}$, and

$$
\begin{aligned}
& \mathbf{S}_{\mathbf{0}}=\frac{1}{n-p-m} \sum_{l=m+1}^{n-p} \widehat{\mathbf{e}}_{(l)+d} \widehat{\mathbf{e}}_{(l)+d}^{\prime} \\
& \mathbf{S}_{\mathbf{1}}=\frac{1}{n-p-m} \sum_{l=m+1}^{n-p} \widehat{\mathbf{w}}_{(l)+d} \widehat{\mathbf{w}}_{(l)+d}^{\prime}
\end{aligned}
$$


where $\widehat{\mathbf{w}}_{t}$ is the least squares residual of regression (2). Under the null hypothesis that $\mathbf{Y}_{t}$ is linear, Tsay (1998) shows that $C(d)$ is asymptotically a chi-squared random variable with $\left(p k^{2}+k\right)$ degrees of freedom.

\section{Model Specification, Estimation and Diagnostic Checking}

To perform the $C(d)$ test for nonlinearity in (3), both values of $p$ and $d$ must be given. In practice, we can select $p$ by the partial autoregression matrix (PAM) of $\mathbf{Y}_{t}$. Tiao and Box (1981) define the PAM at lag $l$, which is denoted by $\Pi(l)$, to be the last matrix coefficient when the data are fitted to a vector autoregressive process of order $l$. This is a direct extension of the Box and Jenkins (1976, p. 64) definition of the partial autocorrelation function for univariate time series. The partial autoregression matrices $\Pi(l)$ of a linear vector $\operatorname{AR}(p)$ process are zero for $l>p$. This "cut-off" property provides very useful information for identifying the order $p$. Once $p$ is selected, $d$ is chosen so that it gives the most significant $C(d)$ statistic.

In univariate TAR modeling, we use various scatterplots for specifying the number of regimes $k$ and the threshold parameters (i.e., the $r$ values). Unfortunately, these plots are not applicable to high dimensional multivariate TAR analysis. Following Tong (1983, p. 186), we use Akaike's Information Criterion (AIC) to search for these parameters.

Given $p, d, k$, and $\mathcal{R}_{k}=\left\{r_{1}, \ldots, r_{k-1}\right\}$, the full-length ordered bivariate autoregression can be divided into regimes. For the $j$ th regime of data, we have a general linear model of the form

$$
\mathbf{Y}_{\mathbf{j}}=\mathbf{A}_{\mathbf{j}} \boldsymbol{\Phi}^{(\mathbf{j})}+\varepsilon_{\mathbf{j}}
$$

where

$$
\begin{aligned}
& \mathbf{Y}_{\mathbf{j}}=\left(\mathbf{Y}_{\left(\pi_{j-1}+1\right)+d}^{\prime}, \mathbf{Y}_{\left(\pi_{j-1}+2\right)+d}^{\prime}, \ldots, \mathbf{Y}_{\left(\pi_{j}\right)+d}^{\prime}\right)^{\prime} \\
& \mathbf{\Phi}^{(\mathbf{j})}=\left(\mathbf{C}_{0}^{\prime}, \mathbf{\Phi}_{1}^{\prime(j)}, \ldots, \mathbf{\Phi}_{p}^{\prime(j)}\right)^{\prime} \\
& \boldsymbol{\varepsilon}_{\mathbf{j}}=\left(\boldsymbol{\varepsilon}_{\left(\pi_{j-1}+1\right)+d}^{\prime}, \boldsymbol{\varepsilon}_{\left(\pi_{j-1}+2\right)+d}^{\prime}, \ldots, \boldsymbol{\varepsilon}_{\left(\pi_{j}\right)+d}^{\prime}\right)^{\prime} \\
& \mathbf{A}_{\mathbf{j}}=\left(\begin{array}{cccc}
1 & \mathbf{Y}_{\left(\pi_{j-1}+1\right)+d-1}^{\prime} & \ldots & \mathbf{Y}_{\left(\pi_{j-1}+1\right)+d-p}^{\prime} \\
1 & \mathbf{Y}_{\left(\pi_{j-1}+2\right)+d-1}^{\prime} & \ldots & \mathbf{Y}_{\left(\pi_{j-1}+2\right)+d-p}^{\prime} \\
\vdots & \vdots & \ddots & \vdots \\
1 & \mathbf{Y}_{\left(\pi_{j}\right)+d-1}^{\prime} & \cdots & \mathbf{Y}_{\left(\pi_{j}\right)+d-p}^{\prime}
\end{array}\right)
\end{aligned}
$$

where $\pi_{j}$ is the largest value of $j$ such that $\left\{r_{j-1}<\right.$ $\left.z_{(j)} \leq r_{j}\right\}$ for $j=1, \ldots, k-1$. We define $\pi_{0}=0$ and $\pi_{k}=n-p$. The number of observations in the $j$ th regime is $n_{j}=\pi_{j}-\pi_{j-1}$. The least squares estimate of $\boldsymbol{\Phi}^{(\mathbf{j})}$ can be obtained by the ordinary multivariate least squares method:

$$
\widehat{\boldsymbol{\Phi}}^{(\mathbf{j})}=\left(\mathbf{A}_{\mathbf{j}}^{\prime} \mathbf{A}_{\mathbf{j}}\right)^{-1}\left(\mathbf{A}_{\mathbf{j}}^{\prime} \mathbf{Y}_{\mathbf{j}}\right) \text {. }
$$

The residual variance-covariance matrix for the $j$ th regime can be obtained by

$$
\widehat{\boldsymbol{\Sigma}}_{j}=\frac{1}{n_{j}} \sum_{t=1}^{n_{j}}\left\{\widehat{\boldsymbol{\varepsilon}}_{\left(\pi_{j-1}+t\right)+d} \widehat{\boldsymbol{\varepsilon}}_{\left(\pi_{j-1}+t\right)+d}^{\prime}\right\} .
$$

The AIC of a bivariate fitted TAR model in (1) is defined as

$\operatorname{AIC}\left(p, d, k, \mathcal{R}_{k}\right)=\sum_{j=1}^{k}\left\{n_{j} \ln \left|\widehat{\boldsymbol{\Sigma}}_{j}\right|+2 k(k p+1)\right\}$.

Given $p$ and $d$, we can search the parameters $k$ and $\mathcal{R}_{k}$ by minimizing the AIC. Due to the computational complexity and possible interpretations of the final model, we usually restrict $k$ to a small number, such as 2 or 3 . For the threshold parameters $\mathcal{R}_{k}$, we divide the data into subgroups according to the empirical percentiles of $z_{t-d}$, and use the AIC to select the $r$ values. Finally, the AIC is used to refine the $\mathrm{AR}$ order $\left(p_{k} \leq p\right)$ in each regime.

To guard against incorrectly specifying the model, a detailed diagnostic analysis of the residuals is required. This includes an examination of the plots of standardized residuals and the sample cross-correlation matrices of the residuals (Tiao and Box, 1981).

\section{EMPIRICAL RESULTS}

In this section, we consider BTAR modeling of monthly Australian interest rates. The series under study are 2-year $\left(x_{1 t}\right)$ and 15-year $\left(x_{2 t}\right)$ government bonds, representing short-term and long-term series in the term structure of interest rates. The data were obtained from the International Monetary Fund (2003). The analysis will be based on the growth series; that is, let $\mathbf{Y}_{t}=\left(y_{1 t}, y_{2 t}\right)^{\prime}$, where $y_{i t}=\ln \left(x_{i t}\right)-\ln \left(x_{i, t-1}\right)$ for $i=1,2$. The time frame of the study is January 1957 to August 2002, with 537 observations of $\mathbf{Y}_{t}$.

Following Tsay (1998), we employ the 3month moving-average "spread" of logged interest rates as the threshold variable. Let $s_{t}=\ln \left(y_{1 t}\right)-$ $\ln \left(y_{2 t}\right)$ be the "spread" at time $t$. The threshold variable $z_{t}$ is defined as

$$
\begin{aligned}
& z_{1}=s_{1}, \quad z_{2}=\left(s_{1}+s_{2}\right) / 2, \\
& z_{t}=\left(s_{t}+s_{t-1}+s_{t-2}\right) / 3, \quad t \geq 3 .
\end{aligned}
$$

Under normal conditions, interest rates are positively correlated with maturities. The correlation between interest rates and maturities may become negative when the economy is in contraction. Thus, the threshold variable $\left(z_{t}\right)$ may be a good proxy for the status of the economy (i.e., negative values of $z_{t}$ indicating an expansion of the economy and positive values signaling a weak economy). Figure 1 plots the $z_{t}$ variable using Australian data. As expected, 
the spread assumed positive values only in the middle of 1970s and the period 1987-1992, when the Australian economy was weak.

We first examine the partial autoregression matrices of the observed vector time series. Tiao and Box (1981) suggest summarizing the PAM using indicator symbols,+- , and $\cdot$, where + denotes a value that is greater than twice the estimated standard error, - denotes a value that is less than twice the estimated standard error, and · denotes an insignificant value based on the above criteria. The resulting indicator matrices for the PAM are given in Table 1. The likelihood ratio statistic, $M(l)$, can be used to test the null hypothesis that a PAM is a zero matrix (i.e., $H_{0}: \boldsymbol{\Pi}(l)=\mathbf{0}$ ). Bartlett (1938) shows that the $M(l)$ statistic is asymptotically $\chi^{2}$ distributed with four degrees of freedom if the null hypothesis is true. From Table 1, we observe that the $M(l)$ statistics drop significantly after $l=9$. This suggests that $p=9$ for the $C(d)$ test for nonlinearity. We perform the $C(d)$ test with $p=9$, $d \leq p$ and $m=125$. The results are given in Table 2.

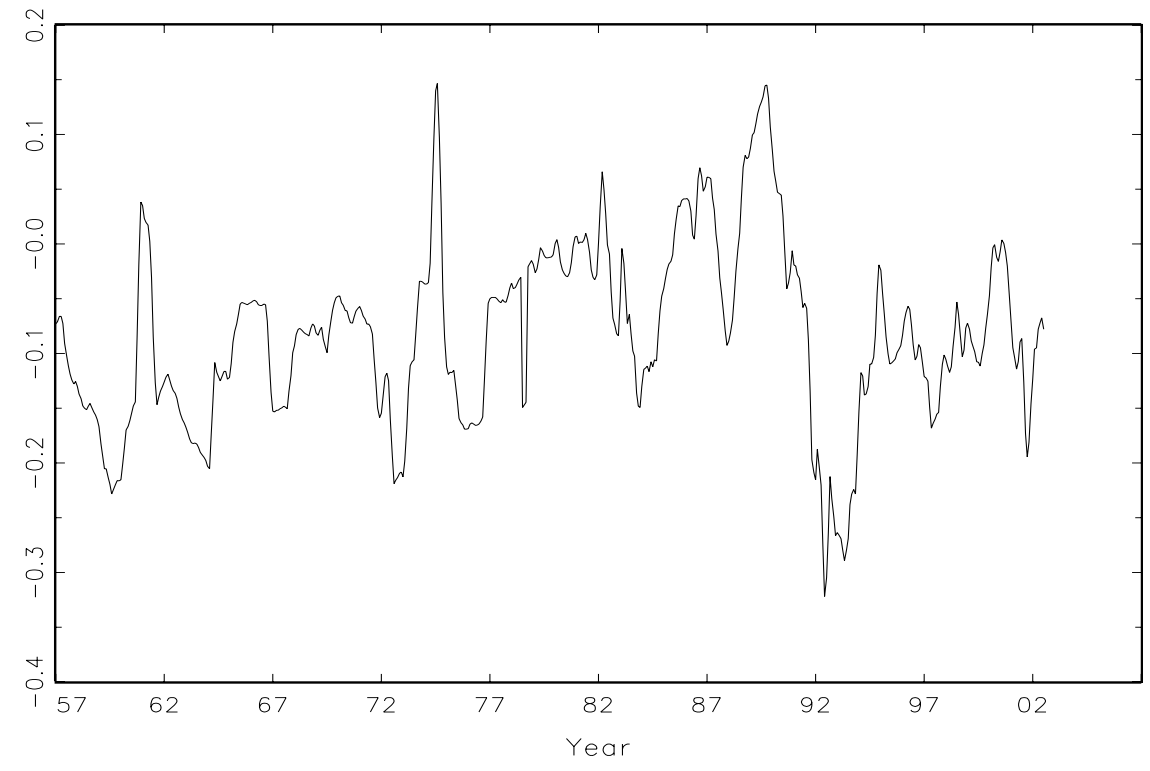

Figure 1. 3-Month moving average of spread in logged Australian interest rates

Table 1. Indicator matrices for the PAM (Australian interest rate data)

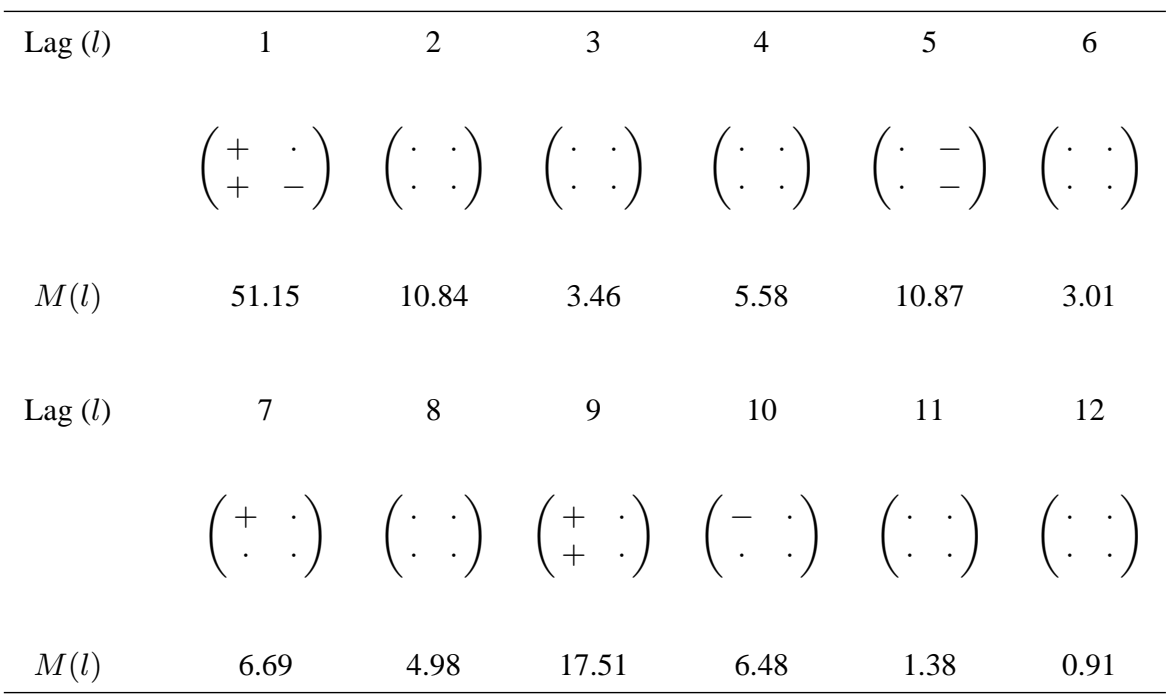

${ }^{*}$ The critical value for the $M(l)$ test is $\chi_{0.95,4}^{2}=9.50$. 
Table 2. Tests for nonlinearity*

\begin{tabular}{cccccc}
\hline$d$ & 1 & 2 & 3 & 4 & 5 \\
$C(d)$ & 119.8 & 95.0 & 76.7 & 76.7 & 76.1 \\
$d$ & 6 & 7 & 8 & 9 & \\
$C(d)$ & 76.3 & 85.7 & 72.8 & 65.4 & \\
\hline
\end{tabular}

The results clearly reject the linear hypothesis. The test statistics also suggest using the delay parameter $d=1$ or $d=2$.

With 547 observations, we only entertain the possibilities of BTAR models with two or three regimes, i.e., $k=2$ or 3 . Given $p, d$, and $k$, we use a grid search method and select the thresholds by minimizing the AIC values that are defined in
(7). Let $\mathcal{P}_{\alpha}\left(z_{t-d}\right)$ be the empirical $\alpha$ th percentile of $z_{t-d}$. For two-regime models, we assume that $r \in\left[\mathcal{P}_{10}\left(z_{t-d}\right), \mathcal{P}_{90}\left(z_{t-d}\right)\right]$. For three-regime models, we assume that $r_{1} \in\left[\mathcal{P}_{10}\left(z_{t-d}\right), \mathcal{P}_{45}\left(z_{t-d}\right)\right]$ and $r_{2} \in\left[\mathcal{P}_{55}\left(z_{t-d}\right), \mathcal{P}_{90}\left(z_{t-d}\right)\right]$. Table 3 shows the selected threshold values under different combinations of $(p, d, k)$. It indicates that the overall minimum AIC is -7292.74 when $k=3, p=$ $9, d=2$ and $\hat{r}_{1}=-0.1457$ and $\hat{r}_{2}=-0.0341$. We further refine the model by allowing different AR orders for different regimes. The AIC selects $\left(p_{1}, p_{2}, p_{3}\right)=(9,5,1)$. The final mininised AIC value is -7334.18 . Least squares estimation results of the specified model are given in Table 4 . The indicator matrices for the residual sample crosscorrelations and the residual PAM are examined, and they do not show any model inadequacy.

Table 3. Selection of $k, p, d$ and threshold values

\begin{tabular}{cccccc}
\hline$k$ & $p$ & $d$ & $\hat{r}_{1}$ & $\hat{r}_{2}$ & AIC \\
\hline 2 & 9 & 1 & -0.1480 & & -7165.50 \\
2 & 9 & 2 & -0.0341 & & -7273.38 \\
3 & 9 & 1 & -0.1481 & -0.0309 & -7215.82 \\
3 & 9 & 2 & -0.1457 & -0.0341 & -7292.74 \\
\hline
\end{tabular}

Table 4. Estimate results

The estimated coefficients $\widehat{\boldsymbol{\Phi}}_{j}^{(k)}$

(a) The first regime $\left(k=1, p_{1}=9\right)$

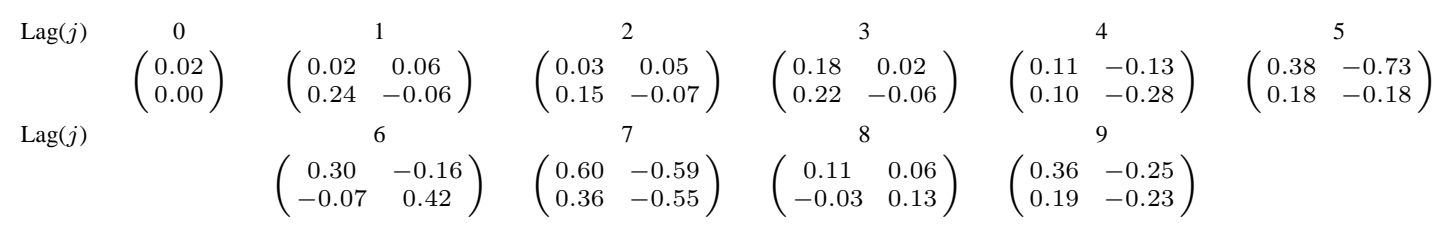

(b) The second regime $\left(k=2, p_{2}=5\right.$ )

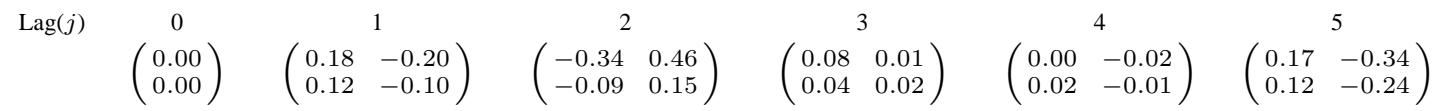

(c) The third regime $\left(k=3, p_{3}=1\right)$

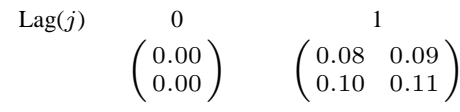




\section{DISCUSSION AND CONCLUSION}

In this paper, we build a BTAR model for analyzing Australian interest rate data. The series under study are 2-year and 15-year government bonds, representing short-term and long-term series in the term structure of interest rates. The 3-month movingaverage spread is employed as the threshold variable. It provides a proxy to the status (expansion versus contraction) of the Australian economy. Our empirical results show that threshold-type nonlinearity clearly exists in the vector series. A BTAR model is fitted. In fact the final model has an economic implication which can be explained easily. In regime 1 , which presents economic expansion periods with short-term interest rate much lower than the long-term rate, the two interest growth series have longer interaction memory $\left(p_{1}=9\right.$ in the final BTAR model). In regime 2 , which represents stable economy, their interaction memory maintains at a moderate level $\left(p_{2}=5\right)$. In regime 3 , which represents economic slowdown or recession, the interest growth rate process has a much shorter memory $\left(p_{3}=1\right)$.

Due to significant financial deregulation that occurred in Australia in the 1970s and 1980s, it is possible that there has been some structural change in the process. International macroeconomics suggest that the change in the exchange rate regime from a fixed, to pegged regime and then to a floating regime is also likely to have some impact on the interest rate determination mechanism. In order to study the robustness of the results in this paper, we apply the BTAR modelling procedures to three sub-periods: (a) January 1957 to December 1969 ( $n=155$ ), (b) January 1970 to December 1989 ( $n=240)$, and (c) January 1990 to August 2002 ( $n=142$ ). BTAR-type nonlinearity is found in each sub-period. However, the optimal lag, delay and autoregressive parameters are different from sub-period to sub-period.

\section{REFERENCES}

Bartlett, M. S., Further aspects of the theory of multiple regression, Proceedings of the Cambridge Philosophical Society, 34, 33-40, 1938.

Box, G.E.P., and G.M. Jenkins, Time Series Analysis: Forecasting and Control, 2nd ed. Holden-Day, San Francisco, 1976.

Chappell, D., J. Padmore, P. Mistry, and C. Ellis, A threshold model for the French Franc/
Deutschmark exchange rate, Journal of Forecasting 15, 155-164, 1996.

De Gooijer, J.G., and Vidiella-i-Anguera, A., Nonlinear stochastic inflation modelling using SEASETARs, Insurance: Mathematics and Economics 809, 116, 2003.

International Monetary Fund, International Financial Statistics, CD-ROM Edition, 2003.

Kräger, H., and P. Kugler, Nonlinearities in foreign exchange markets: a different perspective, Journal of International Money and Finance 12, 195-208, 1993.

Montgomery, A., V. Zarnowitz, R.S. Tsay, and G. Tiao, Forecasting the U.S. unemployment rate Journal of the American Statistical Association 93, 478493, 1998.

Peel, D.A., and A. Speight, Testing for nonlinear dependence in inter-war exchange rates, Weltwirtschaftsliches Archiv 130, 391-417, 1994.

Potter, S., A nonlinear approach to U.S. GNP. Journal of Applied Econometrics 10, 109-125, 1995.

Tiao, G. C., and G.E.P. Box, Modeling multiple time series with applications, Journal of the American Statistical Association 76: 802-816, 1981.

Tiao, G., and R.S. Tsay, Some advances in non-linear and adaptive modelling in time-series, Journal of Forecasting 13, 109-131, 1994.

Timm N. H., and T.A. Mieczkowski, Univariate and Multivariate General Linear Models: Theory and Applications Using SAS Software, SAS Publishing, North Carolina, 1997.

Tong, H., On a Threshold Model, in Pattern Recognition and Signal Processing, edited by Chen, C. H., Sijhoff and Noordoff, Amsterdam, 1978.

Tong, H., Threshold Models in Non-Linear Time Series Analysis, Springer-Verlag, New York, 1983.

Tsay, R. S., Testing and modelling threshold autoregressive processes, Journal of the American Statistical Association 84, 231-240, 1989.

Tsay, R. S., Testing and modelling multivariate threshold models, Journal of the American Statistical Association 93, 1188-1202, 1998.

Wilkie, A.D., More on a stochastic asset model for actuarial use, British Actuarial Journal 1, 777-964, 1995.

Yadav, P.K., P.F. Pope, and K. Paudyal, Threshold autoregressive modelling in finance: the price difference of equivalent assets, Mathematical Finance 4, 205-221, 1994. 